Journal of Computer Science 5 (9): 630-634, 2009

ISSN 1549-3636

(C) 2009 Science Publications

\title{
Throughput-Delay Trade-Off for Slotted Aloha Multiple Access with Capture Effect
}

\author{
${ }^{1,2}$ A. Chehri, ${ }^{2}$ P. Fortier and ${ }^{2}$ P.M. Tardif \\ ${ }^{1}$ Underground Communications Research Laboratory, \\ 3rd Avenue Local 103, Val-d'Or, J9P 1S2, Québec, Canada \\ ${ }^{2}$ Department of Electrical and Computer Engineering, Laval University, Québec, Canada
}

\begin{abstract}
Problem statement: Medium Access Control (MAC) protocols are one of most important research issues in communication and networking. There have been proposed several MAC protocols in wireless as well in wired networks. Approach: ALOHA-based protocol is the simplest one, being able to provide prompt access, reliable channels and support for quality of service. However its limited capacity, low throughput and excessive delays make it not suitable for several applications. Results: So there had been many efforts devoted to increase its performance, one of them is the capture effect. With capture effect, the packets arriving with the highest power had a good chance to be detected accurately, even when other packets were present. Conclusion: In this study, a throughput-delay tradeoff was investigated to improve the network performance. The capture threshold was assigned to the highest throughout/time delay ratio. Therefore, the capture threshold which provided a high throughput and low time delay was selected by a central node.
\end{abstract}

Key words: Slotted ALOHA, outage probability, throughput, time delay, capture effect

\section{INTRODUCTION}

Multiple Access Protocols (MAC) is an active research area form several years and there exist a huge body in literature. Some survey study covering MAC protocols in general as well as wireless MAC protocols can be found $\mathrm{in}^{[1,2]}$. Traditionally, the most important performance requirement for MAC protocols are throughput efficiency, stability, fairness, low access delay (time between packet arrival and the first attempt to transmit it and low transmission delay (time between packet arrival and successful delivery), as well as low overhead.

Among all MAC protocols, random Medium Access Control (MAC) protocols have been widely studied for wireless networks due to their low cost and easy implementation. Aloha is a fundamental technique for multiple access communication and from the basis of a number of major protocols in modern communications CSMA, ISMA. ALOHA is arguably the simplest, are appropriate for sharing a channel among a large population of users with busty traffic ${ }^{[3]}$.

Aloha was first introduced by Abramson ${ }^{[4]}$, where it was determined that the maximum channel utilization under "pure Aloha" is about 18\% of the channel bandwidth. The slotted variation was then introduced by Roberts, showing its capacity is doubled (over pure Aloha), to about $36 \%{ }^{[5]}$.

In classical analyses of slotted ALOHA networks, it was assumed that all packets arriving at the central node with the same power and all packets involved in a collision are destroyed ${ }^{[6]}$. This is a reasonable assumption when all the packets are received under nearly equal power levels. However, this assumption is somewhat not realistic in a wireless radio environment, where the received packets at the central node are subject to the near/far effect, propagation path loss, the effects of shadowing, the position of transmitter, which cause differences in received power levels. This phenomenon gives rise to the capture effect ${ }^{[6]}$ : The packet arriving with the highest energy has a good chance of being detected accurately, even when other packets are present. In a real communication system, the central node decides whether the packet generated form the transmitter nodes are successfully transmitted and the results of the decision are transmitted to the concerned node.

In this study, the capture is defined in a more abstract way as a situation where two or more nodes with different levels execute transmissions in parallel and the transmitter node with the strong level succeeds in transmission without being affected by other nodes.

Corresponding Author: A. Chehri, Underground Communications Research Laboratory, 3rd Avenue Local 103, Val-d'Or, J9P 1S2, Québec, Canada 
While there is a rich body of literature addressing this problem, this topic is far from being mature.

In this context, we investigate the capture effect in slotted Aloha for a typical wireless ad hoc or sensor networks in confined areas. A suitable capture threshold was evaluated to increase the performance of the networks. A scalar function was assigned from many Capture Thresholds (CTs) and only one CT was selected by the central node. The goals are the get a highest throughput with a lowest time delay. Hence, the nodes are able to get a high capacity to communication with central node and can transmit their packets with low time.

\section{MATERIALS AND METHODS}

Model specifications and assumptions: We consider ad hoc networks with $\mathrm{N}$ nodes trying to transmit data to central node. Each node has an infinite buffer memory which is used to store the generated date until they are sent successfully to the central node. The incoming packets are all assumed to be the same length. Each node is assumed to generate packets randomly and independently. Such packet generation is follows Poisson distribution and each one is independent of the pervious packet generation. The size of each packet is fixed; we assume also that the probability of packet does not change in each time slot of the simulation.

The transmitted power of each packet is the same of all nodes hence no power control is considered. Therefore the received power of each packet is depend on the position of the node.

The received power of each packet is dependent on the position of the sensor and the condition of the communication channel. Therefore, even if several packets come into collision, the packet that has the largest received power sometimes survives. On the other hand, even if a collision does not occur, a packet transmission error occurs because the received power at the clusterhead is smaller than the required power when the condition of the communication channel is worse.

The radio channel can be characterized statistically by the multipath fading and large scale model. In fact, the wireless communication system, the quality of the communication channel is time-variant, the propagation depend on the distance between transmitter and receiver with path loss exponent $\alpha$. Shadowing exhibits timevarying factors caused by diffraction of traveling waves around large objects such as large obstacles. It is added on top of the path loss and represented as a random fluctuation with a log-normal distribution, with a standard deviation $\sigma$.

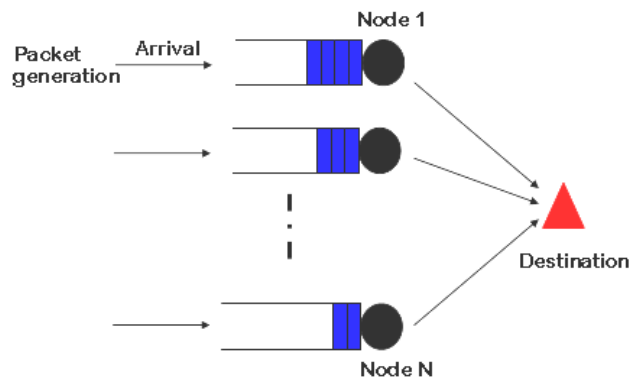

Fig. 1: The scheduling scheme viewed as a queuing system

We consider the network topology whereby the central node is positioned in the center of the area. A variable number of source nodes are randomly distributed throughout the network area depending on it topology and within a single transmission hop to the central node (Fig. 1).

In this study we define the total packets (transmitted, generated and even retransmitted) to the central node as the offered traffic.

Two performances are evaluated: the throughput which is defined as the average number of successfully received packets per time slot. If the transmission data rate, quantity of information in a packet and number of packets successfully transmitted are defined as $\mathrm{R}(\mathrm{bps}), \mathrm{T}(\mathrm{bit})$ and $\mathrm{n}$ respectively. The normalized throughput is given as ${ }^{[3]}$ :

$\mathrm{S}=\frac{\mathrm{T} \times \mathrm{n}}{\mathrm{R}}$

If no transmission packets are generated or all transmission packets are destroyed by collisions, S equal to 0 . The ideal characteristic of normalized throughput is when all transmitted packets are received perfectly; the value of $\mathrm{S}$ becomes equal to 1 .

The second parameter is the Transmission Delay (TD). TD is defined as the time duration of a packet lasts in the system since it is being handled by the slotted aloha layer until the reception of acknowledgement of its successful reception. The average transmission delay is dependent on the length of packet.

This is where it is determined whether the transmitted packets from transmitter node are successfully received at the central node. The average transmission delay is dependent on the length of packet.

\section{RESULTS AND DISCUSSION}

In this study, we study the performance of slotted aloha in particular environment (underground mines). 
In fact since few decades a large companies has investigate to deploy ad hoc network in underground mines. Among all ad hoc networks that can be deployed in underground mines we can cote sensor and actuator networks, mesh networks. Their goals are generally to increase the productivity and decrease the cost installation with eliminating the costly wired equipments.

In this context, we investigate in deep the capture effect in slotted aloha for a typical ad hoc or sensor networks. A custom-made simulation tool was developed. In order to simulate the positioning scheme involving these nodes it is necessary to establish a network from scratch. In addition to transmitters and receivers, a channel model will have to be simulated in order to obtain results that are as realistic as possible.

Algorithm 1: Application of slotted aloha with capture effect for ad hoc multiple accesses in confined area:

$\%$ Position of central node $(\mathrm{X}, \mathrm{Y}, \mathrm{Z})$,

$\%$ Number of node $\mathrm{N}$

$\%$ Position of nodes $\left(\mathrm{X}_{\mathrm{i}}, \mathrm{Y}_{\mathrm{i}}, \mathrm{Z}_{\mathrm{i}}\right)$;

$\%$ Capture effect threshold.

$\%$ Number of transmitted packet,

$\%$ Wireless underground mines channel model.

$\%$ Traffic model (packet generation)

1: While stopping criterion not met (number of packet), do:

1: Evaluate the access protocol.

1. Decision whether the transmission packet is successfully transmitted to central node.

(a) Measurement of transmission delay.

(b) Measurement of number of successfully transmitted packet

\section{2: End while}

3: Output: throughput and measurement delay

$\%$ end of simulation

As mentioned early, by the capture effect, the packets with maximum received power may survive at the central node. Then, the CT is calculated at the central node for all packets that are transmitted from all nodes and the packet that obtains the maximum CT is selected.

Then, for the selected packet, the instantaneous CT is calculated again by regarding the other packets as noise. If the calculated $\mathrm{C} / \mathrm{N}$ is larger than determined threshold $\gamma_{0}$, the instantaneous data in the packet is transmitted to the cluster-head point successfully and the result is stored. On the other hand, if the calculated
$\mathrm{C} / \mathrm{N}$ is lower than the threshold $\gamma_{0}$, all packets from nodes are not transmitted correctly to the cluster-head node. A collision message will be send to these corresponding nodes.

The model of wireless channel presented in this study is described $i^{[7]}$. Since other details of the propagation experiments and modelling are given $\mathrm{in}^{[7]}$, we are going to summarize here only the fundamental issues. Their channel was modulated using the measurements which were made in an experimental underground mine.

The central frequency of $2.4 \mathrm{GHz}$ was selected in order to ensure compatibility with Wireless Local Area Network (WLAN) systems and ad hoc networks. The author finds the value of the path-loss exponent is equal to 2.16 with a standard deviation $(\sigma)$ of $6.13 \mathrm{~dB}$.

20 nodes are randomly deployed in underground mine gallery. The topology of networks in evaluated using a virtual mine, an example of virtual deployment of wireless node is shown in Fig. 2.

The maximal range between node and clusterhead (central node) is fixed to $40 \mathrm{~m}$.

At the clusterhead are able to adapt the capture widow form $0-20 \mathrm{~dB}$. The rate is assumed to be $512 \mathrm{kbits} \mathrm{sec}^{-1}$ and the packet length is fixed 128 bits.

As shown in Fig. 3, when the capture effect threshold is great, the throughput is close to the theatrical value $(38 \%)$. Moreover, if the capture effect is considered, a transmitted packet sometimes survived because the received powers for arrival packets were different for each other (the distance to the central node are different form the transmitted nodes).

Therefore, the throughput increased. Moreover, the throughput increases when the capture effect threshold decreases.

In addition, the average transmission delay is reduced with the capture effect as shown in Fig. 4.

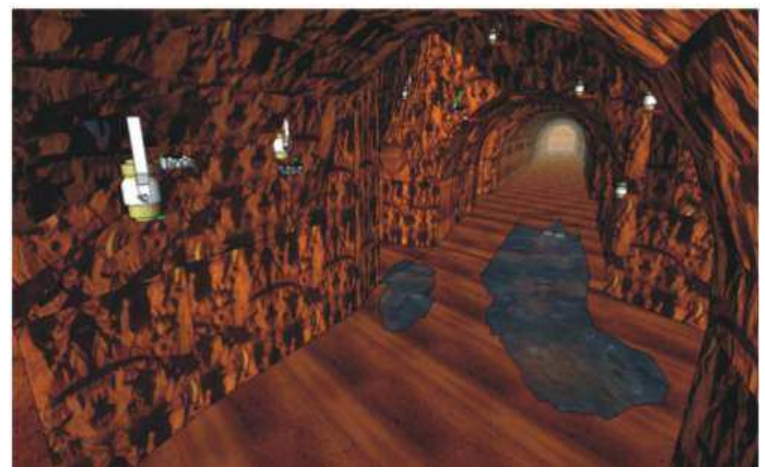

Fig. 2: Example of deployment of ad hoc networks in underground mines 


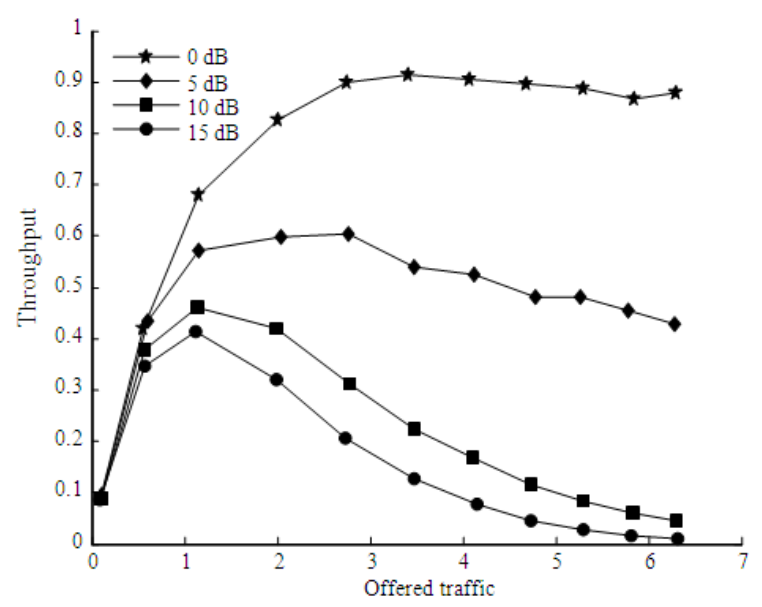

Fig. 3: Throughput of slotted aloha with capture effect

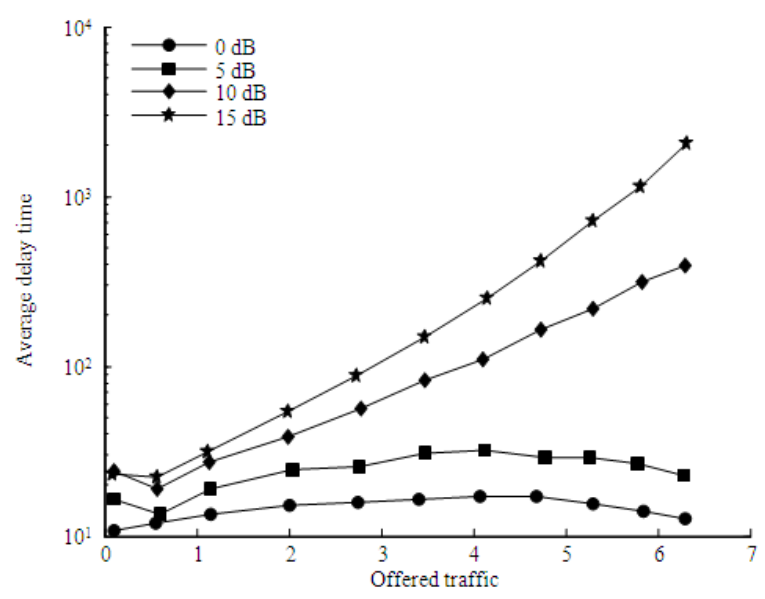

Fig. 4: Averaged delay time of slotted aloha with capture effect

Throughput-delay trade-off analysis: As we have seen the capture effect increases the performance of the system. However, what is the capture threshold in the kind of environment to produce the highest performance.

In this part we described a suitable threshold to increase the throughput with lower time delay packet transmission. We consider the problem of minimizing a cost function based on both parameters.

$F(\gamma)=\frac{S}{D}$

where, $\mathrm{S}$ and $\mathrm{D}$ are the normalized throughput and time delay packet transmission.

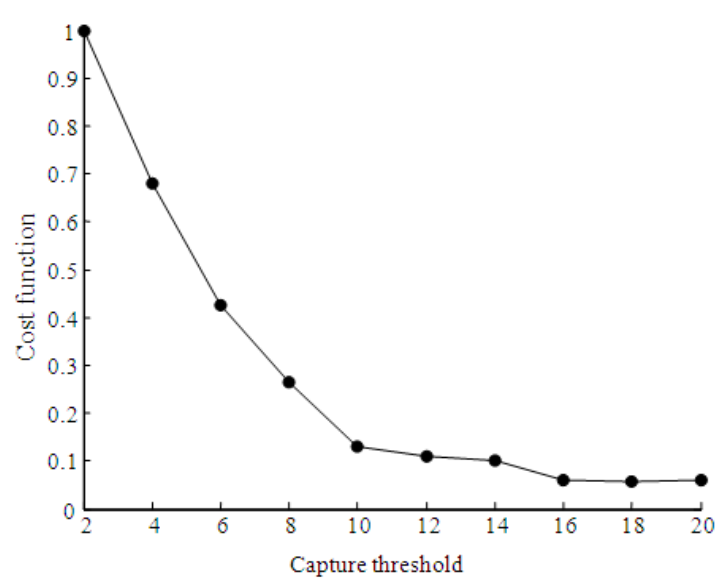

Fig. 5: The normalized cost function with capture effect

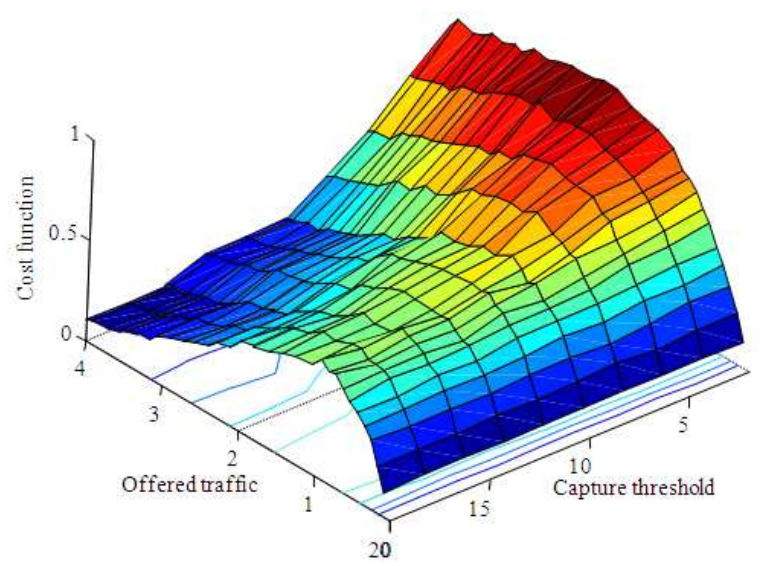

Fig. 6: The normalized cost functions with capture effect and offered traffic

The optimal capture threshold is obtained by minimizing the cost function $F(\gamma)$ given in (2). To determinate the estimated location many scenario algorithms have been simulated.

Figure 5 shows the normalized cost function of model (2) as function of different value of Capture Threshold (CT). The values of CT to get the best performance of the networks (on the term of high throughput with low average delay time) are corresponding to the lowest values (less than $10 \mathrm{~dB}$ ). Figure 6 shows the normalized cost function as function of the offered traffic and the capture threshold. The figure shows also that the lowest values of TC are the most suitable values to get the best performance.

\section{CONLUSION}

In this study, we have studied the performance of slotted aloha with capture effect for wireless networks. 
The analyses are investigated in particular environment (underground mines). We have investigated on the throughput-delay trade-off in under to improve the performance of the networks.

A cost function is assigned to the throughout/average time delay ratio; the goad was maximizing this function. The capture threshold which provide a high throughput and low time delay is selected by a central node. The value of CT is often corresponding to $10 \mathrm{~dB}$ or less.

\section{ACKNOWLEDGEMENT}

This research was done with support of University of Quebec in Abitibi Temiscamingue (UQAT). The author would like to acknowledge the continuing guidance of LRCS laboratory.

\section{REFERENCES}

1. Akyldiz, I.F., J. McNair, L.C. Martorell, R. Puigjaner and Y. Yesha, 1999. Medium access control protocols for multimedia traffic in wireless networks. IEEE. Network, 13: 39-47. DOI: 10.1109/65.777440

2. Holger, K. and A. Willig, 2005. Protocols and Architectures for Wireless Sensor Networks. John Wiley and Sons, Ltd., ISBN: 0470095105, pp: 497.
3. Harada, H. and R. Prasad, 2002. Simulation and Software Radio for Mobile Communications. Artech. House, Boston/London, ISBN: 1580530443 , pp: 467.

4. Abramson, N., 1970. The aloha system-another alternative for computer communications. Proceedings of the Fall Joint Computer Conference, Nov. 17-19, ACM Press, Houston, Texas, pp: 281-285. http://portal.acm.org/citation.cfm?id=1478502.

5. Roberts, L., 1975. Aloha packet system with and without slots and capture. Comput. Commun. Rev., 5: 28-42.

http://portal.acm.org/citation.cfm?id=1024920

6. Behzad, A., I. Rubin and J. Hsu, 2005. On the performance of randomized power control algorithms in multiple access wireless networks. Proceedings of the IEEE Wireless Communications and Networking Conference, Mar. 13-17, IEEE Xplore Press, USA., pp: 707-711. DOI: 10.1109/WCNC.2005.1424594

7. Nerguizian, C., C.L. Despins, S. Affes and M. Djadel, 2005. Radio-channel characterization of an underground mine at $2.4 \mathrm{Ghz}$. IEEE. Trans. Wireless Commun., 4: 2441-2453. DOI: 10.1109/TWC.2005.853899 